\title{
Low Predictive Value of FRAX Adjusted by Trabecular Bone Score for Osteoporotic Fractures in Korean Women: A Community-Based Cohort Study
}

\author{
Hana Kim ${ }^{1,2, *}$, Jung Hee Kim ${ }^{1, *}$, Min Joo Kim ${ }^{1}$, A Ram Hong ${ }^{1}$, HyungJin Choi ${ }^{3}$, EuJeong Ku ${ }^{4}$, Ji Hyun Lee \\ Chan Soo Shin ${ }^{1}$, Nam H. Cho ${ }^{6}$ \\ ${ }^{1}$ Department of Internal Medicine, Seoul National University College of Medicine, Seoul; ${ }^{2}$ Department of Internal Medicine, \\ Ilsan Cha Medical Center, Goyang; ${ }^{3}$ Department of Anatomy, Seoul National University College of Medicine, Seoul; \\ ${ }^{4}$ Department of Internal Medicine, Chungbuk National University College of Medicine, Cheongju; ${ }^{5}$ Department of Internal \\ Medicine, Veterans Health Service Medical Center, Seoul; ${ }^{6}$ Department of Preventive Medicine, Ajou University School of \\ Medicine, Suwon, Korea
}

Background: The value of the Fracture Risk Assessment Tool (FRAX) and the trabecular bone score (TBS) for assessing osteoporotic fracture risk has not been fully elucidated in Koreans. We conducted this study to clarify the predictive value of FRAX adjusted by TBS for osteoporotic fractures in Korean women.

Methods: After screening 7,192 eligible subjects from the Ansung cohort, 1,165 women aged 45 to 76 years with available bone mineral density (BMD) and TBS data were enrolled in this study. We assessed their clinical risk factors for osteoporotic fractures and evaluated the predictive value of FRAX with or without BMD and TBS.

Results: During the mean follow-up period of 7.5 years, 99 (8.5\%) women suffered major osteoporotic fractures (MOFs) and 28 (2.4\%) experienced hip fractures. FRAX without BMD, BMD-adjusted FRAX, and TBS-adjusted FRAX were significantly associated with the risk of MOFs (hazard ratio [HR] per percent increase, 1.08; 95\% confidence interval [CI], 1.03 to 1.14; HR, 1.09; 95\% CI, 1.03 to 1.15; and HR, 1.07; 95\% CI, 1.02 to 1.13, respectively). However, BMD-adjusted FRAX and TBS-adjusted FRAX did not predict MOFs better than FRAX without BMD based on the Harrell's C statistic. FRAX probabilities showed limited value for predicting hip fractures. The cut-off values of FRAX without BMD, FRAX with BMD, and FRAX with BMD adjusted by TBS for predicting MOFs were $7.2 \%, 5.0 \%$, and $6.7 \%$, respectively.

Conclusion: FRAX with BMD and TBS adjustment did not show better predictive value for osteoporotic fractures in this study than FRAX without adjustment. Moreover, the cut-off values of FRAX probabilities for treatment might be lower in Korean women than in other countries.

Keywords: Osteoporosis; Osteoporotic fracture; Risk assessment; Bone density; Fracture risk assessment tool; Trabecular bone score

Received: 13 February 2020, Revised: 30 March 2020,

Accepted: 14 April 2020

Corresponding author: Nam H. Cho

Department of Preventive Medicine, Ajou University School of Medicine, 164 World cup-ro, Yeongtong-gu, Suwon 16499, Korea

Tel: +82-31-219-5900, Fax: +82-31-219-5901, E-mail: chnaha@ajou.ac.kr
Copyright $\odot 2020$ Korean Endocrine Society

This is an Open Access article distributed under the terms of the Creative Commons Attribution Non-Commercial License (https://creativecommons.org/ licenses/by-nc/4.0/) which permits unrestricted non-commercial use, distribution, and reproduction in any medium, provided the original work is properly cited.

*These authors contributed equally to this work. 


\section{INTRODUCTION}

Osteoporosis is defined as a fragile bone status characterized by low bone mass, bone microarchitecture deterioration, and reduced bone strength, which increase the risk of fragility fractures [1]. Previous studies reported that mortality significantly increased after osteoporotic vertebral and hip fractures [2-4]. In Korea, the risk of experiencing osteoporotic fractures during the remaining lifetime in persons older than 50 years was estimated to be $23.8 \%$ for men and $59.5 \%$ for women [5]. Thus, the precise prediction of osteoporotic fractures would be helpful to reduce their consequent burden.

Bone mineral density (BMD) measured using dual-energy Xray absorptiometry (DXA) is known to be a major determinant of osteoporotic fracture risk [6]. However, BMD provides little information about bone quality, including the bone microarchitecture. Furthermore, severe multiple vertebral compression fractures, degenerative changes, or aortic calcification can cause a false increase in spinal BMD [7]. Moreover, previous studies reported that most fragility fractures occurred in people with normal or osteopenic BMD values [8,9]. Recently, the lumbar spine trabecular bone score (TBS) was introduced as a measurement of bone texture that is correlated with fracture risk independently of BMD [10-12]. Several studies have reported that a low TBS was correlated with an increased fracture risk [13-15].

Clinical risk factors such as parental history of hip fracture, exposure to systemic glucocorticoids, history of prior fragility fracture, current smoking, high alcohol intake, and the presence of rheumatoid arthritis play a pivotal role in the assessment of osteoporotic fracture risk [16-20]. Although several fracture risk prediction tools based on clinical risk factors have been created, the Fracture Risk Assessment Tool (FRAX), which was developed in 2008 based on large prospective cohort studies, is widely used worldwide [21]. However, the risk factors and incidence of osteoporotic fractures vary widely among populations [22] and the incidence of osteoporotic fractures in Korea is known to be lower than that in Western countries [23,24]. The calculated FRAX probability was also lower in Koreans than in Western populations $[25,26]$. Thus, population-specific data on the treatment threshold for FRAX are required to predict the risk of fracture. Moreover, the value of the recently introduced TBSadjusted FRAX for predicting osteoporotic fractures has not been validated in Koreans.

We aimed to evaluate the predictive value of the TBS-adjusted FRAX for osteoporotic fractures, and to suggest cut-off values of FRAX probabilities for osteoporosis treatment in this community-based cohort study of Korean women.

\section{METHODS}

\section{Study population}

The Ansung cohort was established for the Korean Genome and Epidemiology Study in 2001 to investigate the prevalence and determinants of chronic diseases in Korea. This is an ongoing prospective study involving biennial examinations. In Ansung, sampling was based on a clustering sampling method using mailing, door-to-door visits, and telephone requests within five randomly selected local townships (termed myeon in Korea) in the district. After screening 7,192 eligible subjects, a total of 5,018 subjects (2,239 men and 2,279 women) aged between 40 and 69 years were recruited in 2001. Finally, a total of 1,165 women aged 45 to 76 years who voluntarily consented to undergo BMD and TBS measurements during 2007 to 2008 were enrolled because TBS has been available since 2007. The period encompassed by the study extended until 2015 to 2016, and the mean follow-up period was 7.5 years. Forty subjects died during this period. Clinical risk factors for osteoporotic fractures were investigated using self-administered questionnaires, which gathered information on age, menopausal status, history of prior osteoporotic fracture, parental history of hip fragility fracture, smoking status, alcohol consumption, and comorbidities. Smoking status was categorized as never, previous, or current. Alcohol intake history was assessed including the types and quantity of alcohol. All data were collected by trained staff using standardized questionnaires through face-to-face interviews. The questionnaires collected information on all types of arthritis without distinguishing rheumatoid arthritis from osteoarthritis.

The study was approved by the Institutional Review Board of Ajou University Hospital and written informed consent was obtained from all patients (AJIRB-CRO-07-012, AJIRB-MEDSUR-13-016, AJIRB-BMR-SUR-15-179, AJIRB-BMR-SUR16-121).

\section{Assessment of osteoporotic fractures}

The subjects were contacted every 2 years and asked whether they had a new fracture through the self-questionnaire. We included only incident osteoporotic fractures of the spine, hip, humerus, and wrist as major osteoporotic fractures (MOFs). Osteoporotic fractures were defined as fragility fractures that occurred without major trauma or fractures due to a fall from a height lower than the subject's height. We confirmed osteoporotic fractures only through the self-reported questionnaire be- 
cause the time of occurrence of vertebral fractures observed on radiography without any medical history was unclear, and we could not assess osteoporotic fractures immediately during the follow-up interval.

\section{Measurements of BMD and TBS}

Lumbar spine BMD and femoral neck BMD were measured using DXA (Lunar Prodigy, General Electric Medical Systems, Chicago, IL, USA) and analyzed (Encore Software version 11.0, Minneapolis, MN, USA) according to the manufacturer's instructions. When the lumbar spine BMD (L1-L4) was not appropriate because of a compression fracture or severe sclerosis, we used the mean BMD value of the remaining lumbar spine. All TBS values were retrospectively measured with iNsight software version 2.0.0.1 (MedImaps, Geneva, Switzerland), using spine DXA files from the database to ensure that the investigators were blinded to all clinical parameters and outcomes. The software used the raw DXA image of the anteroposterior spine with the same region of interest as that used for the BMD measurements. The instruments were calibrated using anthropomorphic phantoms.

\section{FRAX and TBS-adjusted FRAX calculations}

The FRAX without BMD (including age, sex, body weight, height, history of prior osteoporotic fracture, parental history of hip fragility fracture, current smoking, arthritis, and alcohol consumption $>3$ units/day), FRAX with BMD, and TBS-adjusted FRAX probabilities for MOFs and hip fractures were calculated using the tool for Koreans provided on the FRAX website (https://www.sheffield.ac.uk/FRAX/index.aspx).

\section{Statistical analysis}

Data were given as mean \pm standard deviation (SD) or number $(\%)$. We performed the chi-square test for categorical variables and the Student's $t$ test for continuous variables. Hazard ratios (HRs) with 95\% confidence intervals (CIs) for the time to incident fracture were obtained from Cox proportional hazard models using Stata version 14.2 (StataCorp., College Station, TX, USA). The Harrell's C statistic was used to compare the predictive capability of FRAX without BMD, FRAX with BMD, and TBS-adjusted FRAX probabilities. To evaluate the additional contribution of TBS to the prediction of osteoporotic fractures in osteopenic subjects, we conducted a subgroup analysis. The cut-off value of each FRAX probability was calculated using the 'maximally selected rank statistics' (maxstat) method through the 'maxstat' and 'rpart' packages of $\mathrm{R}$ version 4.3.2 (R
Foundation for Statistical Computing, Vienna, Austria) [27]. The details of this method were described by Hothorn and Lausen $[27,28]$. Briefly, this method assesses all of the data points from the continuous FRAX probability data and establishes a cut-off point where the standardized statistics take their maximum significance for the separation of subjects at a high risk for osteoporotic fracture.

\section{RESULTS}

The baseline characteristics of the study subjects are shown in Table 1 . The mean age \pm SD was $61.6 \pm 8.7$ years. The study subjects comprised 1,072 (92.0\%) postmenopausal women. In total, 123 women $(10.6 \%)$ had a history of prior osteoporotic fracture. Their lumbar spine BMD, femoral neck BMD, and lumbar spine TBS were $0.957 \pm 0.179,0.800 \pm 0.133$, and $1.363 \pm$

Table 1. Baseline Characteristics of the Study Subjects

\begin{tabular}{|c|c|}
\hline Characteristic & $\begin{array}{l}\text { Women } \\
(n=1,165)\end{array}$ \\
\hline Age, yr & $61.6 \pm 8.7$ \\
\hline Height, $\mathrm{cm}$ & $152.3 \pm 6.1$ \\
\hline Weight, $\mathrm{kg}$ & $57.8 \pm 8.7$ \\
\hline BMI, $\mathrm{kg} / \mathrm{m}^{2}$ & $24.9 \pm 3.4$ \\
\hline Menopause & $1,072(92.0)$ \\
\hline Previous history of osteoporotic fracture & $123(10.6)$ \\
\hline Parental history of hip fracture & 0 \\
\hline Current smoking & $18(1.5)$ \\
\hline$\geq 3$ Alcoholic drinks per day & $17(1.5)$ \\
\hline Arthritis & $28(2.4)$ \\
\hline Lumbar spine T-score & $-1.289 \pm 1.494$ \\
\hline Lumbar spine BMD, $\mathrm{g} / \mathrm{cm}^{2}$ & $0.957 \pm 0.179$ \\
\hline Femur neck T-score & $-0.846 \pm 1.106$ \\
\hline Femur neck BMD, $\mathrm{g} / \mathrm{cm}^{2}$ & $0.800 \pm 0.133$ \\
\hline L1-4 TBS & $1.363 \pm 0.097$ \\
\hline FRAX probability for MOF without BMD, $\%$ & $6.4 \pm 3.5$ \\
\hline FRAX probability for hip fracture without BMD, $\%$ & $1.9 \pm 1.9$ \\
\hline FRAX probability for MOF with BMD, $\%$ & $5.4 \pm 2.9$ \\
\hline FRAX probability for hip fracture with BMD, $\%$ & $1.1 \pm 1.6$ \\
\hline FRAX probability for MOF with BMD and TBS, $\%$ & $5.4 \pm 3.2$ \\
\hline FRAX probability for hip fracture with BMD and TBS, $\%$ & $1.1 \pm 1.6$ \\
\hline
\end{tabular}

Values are expressed as mean \pm standard deviation or number (\%). BMI, body mass index; BMD, bone mineral density; L1-4, lumbar spine level 1-4; TBS, trabecular bone score; MOF, major osteoporotic fracture; FRAX, Fracture Risk Assessment Tool. 
$0.097 \mathrm{~g} / \mathrm{cm}^{2}$, respectively (Table 1). The BMD-adjusted FRAX probability for MOF and hip fracture was 5.4\% $\pm 2.9 \%$ and $1.1 \% \pm 1.6 \%$, respectively. The TBS-adjusted FRAX probability was $5.4 \% \pm 3.2 \%$ for MOF and $1.1 \% \pm 1.6 \%$ for hip fracture.

During the median follow-up period of 7.5 years, $99(8.5 \%)$ women experienced MOFs. The most common fracture site was the wrist ( $n=31,2.7 \%)$, followed by the hip ( $n=28,2.4 \%)$, spine $(n=21,1.8 \%)$, and humerus $(n=19,1.6 \%)$. The women who experienced MOFs were significantly older and were more likely to have a prior history of osteoporotic fracture than those who did not. Furthermore, the women with MOFs showed significantly lower lumbar spine BMD, femoral neck BMD, and TBS than those without MOFs. The FRAX probabilities for MOF and hip fracture were significantly higher in the women who experienced MOFs than in those who did not (Table 2).

In Cox proportional hazard regression models, the significant risk factors for MOFs were age, prior osteoporotic fracture, lumbar spine BMD, femur neck BMD, and lumbar spine TBS. Among these, a history of a prior osteoporotic fracture was the strongest risk factor for MOFs (HR, 3.09; 95\% CI, 1.69 to 5.64). The risk for MOFs significantly increased by $63 \%$ as age increased by 10 years. All types of FRAX probabilities were shown to be related to the risk of MOFs (Table 3). However, in the multivariate analysis, only a history of a prior osteoporotic fracture (HR, 2.30; 95\% CI, 1.22 to 4.36) and the BMD-adjusted FRAX probability (HR, 1.08; 95\% CI, 1.01 to 1.15 ) remained significant risk factors for MOFs (Table 3).

No variables were found to significantly elevate the risk of hip fracture owing to the low incidence of hip fractures. The HRs (95\% CIs) of FRAX probabilities without BMD, with BMD, and with BMD adjusted by TBS for hip fractures were 1.00 (95\% CI, 0.99 to 1.02 ), 1.01 (95\% CI, 0.99 to 1.03 ), and 1.01 (95\% CI, 0.99 to 1.03 ), respectively (data not shown).

We compared the predictive power of each model by using

Table 2. Comparison of Clinical Risk Factors and Bone-Related Parameters According to the Occurrence of Major Osteoporotic Fractures

\begin{tabular}{|c|c|c|c|}
\hline Variable & $\begin{array}{l}\text { Women without MOF } \\
\qquad(n=1,066)\end{array}$ & $\begin{array}{l}\text { Women with MOF } \\
\qquad(n=99)\end{array}$ & $P$ value \\
\hline Age, yr & $61.3 \pm 8.8$ & $64.6 \pm 7.5$ & $<0.001$ \\
\hline Height, $\mathrm{cm}$ & $152.4 \pm 6.1$ & $151.4 \pm 6.2$ & 0.153 \\
\hline Weight, $\mathrm{kg}$ & $57.9 \pm 8.7$ & $57.4 \pm 8.9$ & 0.590 \\
\hline BMI, $\mathrm{kg} / \mathrm{m}^{2}$ & $24.9 \pm 3.4$ & $25.0 \pm 3.4$ & 0.857 \\
\hline Menopause & $976(91.6)$ & $96(97.0)$ & 0.077 \\
\hline Previous history of osteoporotic fracture & $99(9.3)$ & $24(24.2)$ & $<0.001$ \\
\hline Current smoking & $16(1.5)$ & $2(2.0)$ & 0.661 \\
\hline$\geq 3$ Alcoholic drinks per day & $16(1.5)$ & $1(1.0)$ & 1.000 \\
\hline Arthritis & $25(2.3)$ & $3(3.0)$ & 0.726 \\
\hline Lumbar spine T-score & $-1.249 \pm 1.498$ & $-1.723 \pm 1.381$ & 0.003 \\
\hline Lumbar spine BMD, $\mathrm{g} / \mathrm{cm}^{2}$ & $0.961 \pm 0.180$ & $0.904 \pm 0.166$ & 0.003 \\
\hline Femoral neck T-score & $-0.817 \pm 1.117$ & $-1.162 \pm 0.936$ & 0.003 \\
\hline Femoral neck BMD, g/cm² & $0.802 \pm 0.134$ & $0.760 \pm 0.112$ & 0.025 \\
\hline L1-4 TBS & $1.366 \pm 0.097$ & $1.332 \pm 0.088$ & 0.001 \\
\hline FRAX probability for MOF without BMD, $\%$ & $6.3 \pm 3.4$ & $7.9 \pm 3.7$ & $<0.001$ \\
\hline FRAX probability for hip fracture without BMD, $\%$ & $1.8 \pm 1.9$ & $2.5 \pm 1.9$ & 0.001 \\
\hline FRAX probability for MOF with BMD, $\%$ & $5.3 \pm 2.9$ & $6.4 \pm 3.0$ & $<0.001$ \\
\hline FRAX probability for hip fracture with BMD, $\%$ & $1.1 \pm 1.5$ & $1.5 \pm 1.8$ & 0.014 \\
\hline FRAX probability for MOF with BMD and TBS, $\%$ & $5.3 \pm 3.2$ & $6.5 \pm 3.3$ & $<0.001$ \\
\hline FRAX probability for hip fracture with BMD and TBS, $\%$ & $1.1 \pm 1.6$ & $1.5 \pm 1.8$ & 0.012 \\
\hline
\end{tabular}

Values are expressed as mean \pm standard deviation or number (\%).

MOF, major osteoporotic fracture; BMI, body mass index; BMD, bone mineral density; L1-4, lumbar spine level 1-4; TBS, trabecular bone score; FRAX, Fracture Risk Assessment Tool. 
Table 3. Cox Regression Models of Age, BMI, Previous History of Fracture, BMD, TBS, and FRAX Probability for Major Osteoporotic Fracture

\begin{tabular}{lcc}
\hline Variable & Unadjusted & Adjusted \\
\hline Age (per 10-yr increase) & $1.63(1.19-2.24)$ & $1.39(0.87-2.22)$ \\
BMI (per SD decrease) & $0.95(0.89-1.03)$ & $0.94(0.87-1.02)$ \\
Menopause & $1.67(0.52-5.33)$ & $0.67(0.17-2.57)$ \\
Previous osteoporotic fracture & $3.09(1.69-5.64)$ & $2.30(1.22-4.36)$ \\
LS BMD (per SD decrease) & $1.20(1.00-1.44)$ & $1.05(0.79-1.38)$ \\
FN BMD (per SD decrease) & $1.32(1.03-1.69)$ & $0.99(0.66-1.47)$ \\
L1-4 TBS (per SD decrease) & $1.43(1.11-1.84)$ & $1.16(0.83-1.62)$ \\
FRAX probability without BMD, \% & $1.10(1.04-1.17)$ & $1.08(1.01-1.15)$ \\
FRAX probability with BMD, \% & $1.10(1.03-1.18)$ & $1.06(0.98-1.15)$ \\
FRAX probability with BMD and TBS, \% & $1.09(1.02-1.15)$ & NA
\end{tabular}

Values are expressed as hazard ratio (95\% confidence interval). Clinical risk factors (age, BMI, menopause, and previous osteoporotic fracture), LS BMD, FN BMD, and L1-4 TBS were adjusted by each of the other factors. FRAX probability without BMD and FRAX probability with BMD were adjusted by TBS. Unadjusted hazard ratios were calculated by univariate Cox proportional hazard model analysis. Adjusted hazard ratios were calculated by multivariate Cox proportional hazard model analysis.

BMI, body mass index; BMD, bone mineral density; TBS, trabecular bone score; FRAX, Fracture Risk Assessment Tool; SD, standard deviation; LS, lumbar spine; FN, femoral neck; L1-4, lumbar spine level 1-4; NA, not available.

Table 4. Harrell's C Statistics and Cut-off Values of FRAX Probability without BMD, with BMD, and with BMD and TBS for Predicting Major Osteoporotic Fractures

\begin{tabular}{lcc}
\hline Variable & Harrell C & $\begin{array}{c}\text { Cut-off value, } \\
\%\end{array}$ \\
\hline FRAX probability without BMD & $0.717^{\mathrm{a}}$ & 7.2 \\
FRAX probability with BMD & $0.711^{\mathrm{b}}$ & 5.0 \\
FRAX probability with BMD and TBS & $0.697^{\mathrm{c}}$ & 6.7
\end{tabular}

FRAX, Fracture Risk Assessment Tool; BMD, bone mineral density; TBS, trabecular bone score.

$P$ value between ${ }^{\mathrm{a}}$ and ${ }^{\mathrm{b}}=0.356 ; P$ value between ${ }^{\mathrm{b}}$ and ${ }^{\mathrm{c}}=0.137 ; P$ value between ${ }^{\mathrm{c}}$ and ${ }^{\mathrm{a}}=0.936$.

the Harrell's C statistic (Table 4). The FRAX probability with BMD and the TBS-adjusted FRAX probability for MOFs did not predict MOFs better than the FRAX probability without BMD. As the FRAX probabilities were very low in this study, we calculated their cut-off values by using the maxstat method for MOF prediction. The cut-off values of FRAX probabilities without BMD, with BMD, and with BMD adjusted by TBS were $7.2 \%, 5.0 \%$, and $6.7 \%$, respectively (all $P<0.001$ ).

\section{DISCUSSION}

In the present study, we demonstrated that clinical risk factors such as age and prior osteoporotic fractures were significant predictors of MOFs. However, BMD- or TBS-adjusted FRAX probabilities did not show improved predictive value for MOFs compared with the clinical risk factor-based FRAX probability alone in community-dwelling Korean women. In addition, the cut-off value of the FRAX probability without BMD for predicting MOFs was $7.2 \%$, which is much lower than the FRAX probability for MOFs of $20 \%$ that is generally used as a threshold for osteoporosis treatment in Western countries.

The present study demonstrated that the FRAX probability without BMD for MOFs in women was comparable to the BMD- or TBS-adjusted FRAX probability. In previous studies assessing the utility of FRAX, the FRAX probability with BMD predicted osteoporotic fractures as well as a multiple logistic regression model combining clinical risk factors including age, body weight, femoral neck BMD, and prior fracture history in women [29-31]. However, most studies that showed good predictive values of FRAX for osteoporotic fractures were performed in Caucasian populations [32-34]. In fact, insufficient data have been published for validating the predictive power of FRAX probabilities in the Korean population. Despite the limited evidence from previous Korean studies regarding the feasibility of FRAX, prior research has indicated that FRAX probabilities in Koreans underestimated the risk of MOFs compared with those in other ethnicities $[25,26,33]$. Our results imply that FRAX probabilities for Koreans should be readjusted using appropriately weighted risk factors influencing osteoporotic frac- 
tures.

Previous studies have shown that TBS may be of additional value in predicting osteoporotic fractures. A Manitoba cohort study demonstrated that spine TBS and BMD were independent risk factors for osteoporotic fractures, and that the combination of these two measurements was superior to either measurement alone in postmenopausal women [14]. According to another study from the United States that validated the predictive value of FRAX in older men, TBS was independently associated with incident major osteoporotic and hip fractures, and the predictive power of FRAX with BMD after TBS adjustment was significantly improved [35]. Moreover, a meta-analysis of studies including 17,809 men and women from 14 prospective population-based cohorts showed that the gradient of risk (GR) of TBS for MOFs was 1.44 (95\% CI, 1.35 to 1.53) when adjusted for age and time since baseline. After an additional adjustment for FRAX probability with BMD, TBS remained a significant independent predictor of osteoporotic fractures (GR, 1.32; 95\% CI, 1.24 to 1.41 ). Adjusting the FRAX probability by TBS, derived from McCloskey et al. [34], resulted in a small increase in the GR (1.76 [95\% CI, 1.65 to 1.87 ] vs. 1.70 [95\% CI, 1.60 to 1.81$]$ ) [15]. However, we demonstrated that a low TBS increased the risk for osteoporotic fractures, but failed to show an additional prognostic role of TBS-adjusted FRAX in predicting MOFs in Korean women. Ripamonti et al. [36] reported that TBS was not associated with spine fragility fractures in postmenopausal women with osteoporosis, although TBS_-but not spine BMD — was a significant risk factor for spine fragility fractures in postmenopausal women without osteoporosis. In addition, Mirzaei et al. [37] compared the predictive value of three different models using BMD, TBS, and the combination of BMD and TBS for spine fragility fractures in postmenopausal women, and they showed no clinical benefit of TBS in postmenopausal women. Adding TBS to BMD or FRAX neither improved the predictive value of BMD for vertebral fractures nor changed the treatment decisions made based on FRAX [37]. In this study, no additional benefit of TBS adjustment was found for the predictive power of the FRAX with BMD model for MOFs and hip fractures. These results could be attributed to the distinctly lower FRAX probabilities in Koreans than in Caucasian populations or even in Japanese people. The narrow distribution of FRAX probabilities in this study can also explain the small value of TBS-adjusted FRAX for fracture risk assessment. No definitive evidence exists to explain the low FRAX probabilities recorded in this study; however, relatively young subjects were included and both the BMD and TBS values were high in sub- jects regardless of whether they experienced MOFs. Moreover, the clinical risk factors assessed through the self-reported questionnaires might be causally linked to the low FRAX probabilities. Consequentially, we also observed that FRAX probabilities were attenuated after BMD and TBS adjustment in our study.

In the present study, the cut-off value of FRAX probabilities for MOF was $7.2 \%$ and that for hip fracture could not be calculated due to the low incidence of hip fracture. The cut-off point of FRAX 10-year probability requiring treatment in United States is $20 \%$ for MOF and 3\% for hip fracture. In Japan, the cut-off point used as an intervention threshold is $15 \%$ for MOF and $3 \%$ for hip fracture. Compared to these values, our study showed very low cut-off points for MOF. A recent study of the FRAX-based intervention threshold in seven Latin American counties reported that the cut-off points of FRAX calculated with BMD according to age varied from country to country. The cut-off values of FRAX for MOF ranged from 1.5\% to $27.5 \%$ in Argentina, from $3.8 \%$ to $25.2 \%$ in Brazil, from $1.6 \%$ to $20.0 \%$ in Chile, from $0.6 \%$ to $10.2 \%$ in Colombia, from $0.9 \%$ to $13.6 \%$ in Ecuador, from $2.6 \%$ to $20.0 \%$ in Mexico, and from $0.7 \%$ to $22.0 \%$ in Venezuela at the ages of 40 and 90 years, respectively [24]. As previously described, our study subjects were younger (with ages ranging from 45 to 76 years) than the Latin American study subjects, and we did not calculate cut-off points for each age group. Moreover, the low FRAX probabilities, which may have been caused by the possibility of underestimating clinical risk factors, and the relatively high BMD and TBS values in the Korean population might lead to a relatively low intervention threshold of FRAX probabilities.

To the best of our knowledge, this is the first large-prospective cohort study conducted in Koreans for almost a decade to verify the ability of TBS-adjusted FRAX to predict osteoporotic fracture. Another strength of this study is that it suggested cutoff values for each FRAX probability as intervention thresholds.

There are also several limitations of our study. First, its short follow-up period of less than 10 years could be a limitation because FRAX algorithms calculate 10-year osteoporotic fracture probability. As a result, the incidence of MOF might have been unrealistically low in this study. Second, the total number of study subjects was too small to support generalized conclusions. Third, we excluded men $(n=907)$ due to the extremely low incidence of MOFs in men $(n=36,4.0 \%)$, which might have led to selection bias. Fourth, another reason why the incidence of MOFs was low in our study might have been that cases of morphometric vertebral compression fractures with no directly relevant medical history were excluded due to the unclear onset of 
fractures. This exclusion might have caused the incidence of osteoporotic fractures to be underestimated. Lastly, the lack of specific clinical information about underlying illnesses affecting bone health due to inaccuracies in the questionnaires might cause bias. For instance, it was not possible to discriminate rheumatoid arthritis from osteoarthritis. Further large prospective cohort studies with long-term follow-up are warranted to confirm the feasibility of FRAX in the Korean population.

In conclusion, the FRAX probability with BMD adjusted for TBS did not predict MOFs and hip fracture better than the FRAX probability with BMD or the FRAX probability without BMD in Korean women. Lower cut-off values of FRAX probabilities appear to be needed to determine whether osteoporosis treatment is indicated in Korean women.

\section{CONFLICTS OF INTEREST}

No potential conflict of interest relevant to this article was reported.

\section{ACKNOWLEDGMENTS}

The results and conclusions are those of the authors, and no official endorsement by the Ansung cohort study is intended or should be inferred. This work was supported by the Research Program funded by the Korea Centers for Disease Control and Prevention (found 2007-E71003-00, 2008-E71005-00, 2009E71007-00, 2010-E71004-00, 2011-E71008-00, 2012-E7100800, 2013-E71007-00, 2014-E71005-00, 2015-P71002-00, 2016E71002-00, 2017-E71002-00, 2018-E71002-00). This study was presented at $2018 \mathrm{ENDO}$ as a featured poster.

\section{AUTHOR CONTRIBUTIONS}

Conception or design: H.K., J.H.K., M.J.K., A.R.H., H.J.C., E.J.K., J.H.L., C.S.S., N.H.C. Acquisition, analysis, or interpretation of data: H.K., J.H.K., C.S.S. Drafting the work or revising: H.K., J.H.K., N.H.C. Final approval of the manuscript: H.K., J.H.K., N.H.C.

\section{ORCID}

Hana Kim https://orcid.org/0000-0002-9221-2988

Jung Hee Kim https://orcid.org/0000-0003-1932-0234

Nam H. Cho https://orcid.org/0000-0003-4187-0929

\section{REFERENCES}

1. Consensus development conference: diagnosis, prophylaxis, and treatment of osteoporosis. Am J Med 1993;94:646-50.

2. Center JR, Nguyen TV, Schneider D, Sambrook PN, Eisman JA. Mortality after all major types of osteoporotic fracture in men and women: an observational study. Lancet 1999;353: 878-82.

3. Johnell O, Kanis JA, Oden A, Sernbo I, Redlund-Johnell I, Petterson C, et al. Mortality after osteoporotic fractures. Osteoporos Int 2004;15:38-42.

4. Bliuc D, Nguyen ND, Milch VE, Nguyen TV, Eisman JA, Center JR. Mortality risk associated with low-trauma osteoporotic fracture and subsequent fracture in men and women. JAMA 2009;301:513-21.

5. Park C, Ha YC, Jang S, Jang S, Yoon HK, Lee YK. The incidence and residual lifetime risk of osteoporosis-related fractures in Korea. J Bone Miner Metab 2011;29:744-51.

6. Johnell O, Kanis JA, Oden A, Johansson H, De Laet C, Delmas $\mathrm{P}$, et al. Predictive value of BMD for hip and other fractures. J Bone Miner Res 2005;20:1185-94.

7. Theodorou DJ, Theodorou SJ. Dual-energy X-ray absorptiometry in clinical practice: application and interpretation of scans beyond the numbers. Clin Imaging 2002;26:43-9.

8. Kanis JA. Assessment of fracture risk and its application to screening for postmenopausal osteoporosis: synopsis of a WHO report. WHO Study Group. Osteoporos Int 1994;4:36881.

9. Miller PD, Siris ES, Barrett-Connor E, Faulkner KG, Wehren LE, Abbott TA, et al. Prediction of fracture risk in postmenopausal white women with peripheral bone densitometry: evidence from the National Osteoporosis Risk Assessment. J Bone Miner Res 2002;17:2222-30.

10. Hans D, Barthe N, Boutroy S, Pothuaud L, Winzenrieth R, Krieg MA. Correlations between trabecular bone score, measured using anteroposterior dual-energy X-ray absorptiometry acquisition, and 3-dimensional parameters of bone microarchitecture: an experimental study on human cadaver vertebrae. J Clin Densitom 2011;14:302-12.

11. Roux JP, Wegrzyn J, Boutroy S, Bouxsein ML, Hans D, Chapurlat $\mathrm{R}$. The predictive value of trabecular bone score (TBS) on whole lumbar vertebrae mechanics: an ex vivo study. Osteoporos Int 2013;24:2455-60.

12. Muschitz C, Kocijan R, Haschka J, Pahr D, Kaider A, Pietschmann $\mathrm{P}$, et al. TBS reflects trabecular microarchitecture in premenopausal women and men with idiopathic osteopo- 
rosis and low-traumatic fractures. Bone 2015;79:259-66.

13. Su Y, Leung J, Hans D, Aubry-Rozier B, Kwok T. Added clinical use of trabecular bone score to BMD for major osteoporotic fracture prediction in older Chinese people: the Mr. OS and Ms. OS cohort study in Hong Kong. Osteoporos Int 2017;28:151-60.

14. Hans D, Goertzen AL, Krieg MA, Leslie WD. Bone microarchitecture assessed by TBS predicts osteoporotic fractures independent of bone density: the Manitoba study. J Bone Miner Res 2011;26:2762-9.

15. McCloskey EV, Oden A, Harvey NC, Leslie WD, Hans D, Johansson $\mathrm{H}$, et al. A meta-analysis of trabecular bone score in fracture risk prediction and its relationship to FRAX. J Bone Miner Res 2016;31:940-8.

16. Kanis JA, Johansson H, Oden A, Johnell O, De Laet C, Eisman JA, et al. A family history of fracture and fracture risk: a meta-analysis. Bone 2004;35:1029-37.

17. Kanis JA, Johansson H, Oden A, Johnell O, de Laet C, Melton III LJ, et al. A meta-analysis of prior corticosteroid use and fracture risk. J Bone Miner Res 2004;19:893-9.

18. De Laet C, Kanis JA, Oden A, Johanson H, Johnell O, Delmas $\mathrm{P}$, et al. Body mass index as a predictor of fracture risk: a meta-analysis. Osteoporos Int 2005;16:1330-8.

19. Kanis JA, Johnell O, Oden A, Johansson H, De Laet C, Eisman JA, et al. Smoking and fracture risk: a meta-analysis. Osteoporos Int 2005;16:155-62.

20. Kanis JA, Johansson H, Johnell O, Oden A, De Laet C, Eisman JA, et al. Alcohol intake as a risk factor for fracture. Osteoporos Int 2005;16:737-42.

21. Kanis JA; World Health Organization, Centre for Metabolic Bone Diseases. Assessment of osteoporosis at the primary health care level: summary report of a WHO scientific group. Geneva: World Health Organization; 2008.

22. Barrett-Connor E, Siris ES, Wehren LE, Miller PD, Abbott TA, Berger ML, et al. Osteoporosis and fracture risk in women of different ethnic groups. J Bone Miner Res 2005;20:185-94.

23. Kanis JA, Johnell O, De Laet C, Jonsson B, Oden A, Ogelsby AK. International variations in hip fracture probabilities: implications for risk assessment. J Bone Miner Res 2002;17: 1237-44.

24. Kanis JA, Burlet N, Cooper C, Delmas PD, Reginster JY, Borgstrom F, et al. European guidance for the diagnosis and management of osteoporosis in postmenopausal women. Osteoporos Int 2008; 19:399-428.

25. So GY, Park KH, Yoon DH, Ryu JH, Choi YS. Feasibility of FRAX for prediction of osteoporotic vertebral fractures in
Korea. Asian Spine J 2012;6:22-8.

26. Min YK, Lee DY, Park YS, Moon YW, Lim SJ, Lee YK, et al. A FRAX experience in Korea: fracture risk probabilities with a country-specific versus a surrogate model. J Bone Metab 2015;22:113-8.

27. Hothorn T, Lausen B. On maximally selected rank statistics. R News 2002;2:3-5.

28. Hothorn T, Lausen B. On the exact distribution of maximally selected rank statistics. Comput Stat Data Anal 2003;43: 121-37.

29. Hillier TA, Cauley JA, Rizzo JH, Pedula KL, Ensrud KE, Bauer DC, et al. WHO absolute fracture risk models (FRAX): do clinical risk factors improve fracture prediction in older women without osteoporosis? J Bone Miner Res 2011;26:1774-82.

30. Tamaki J, Iki M, Kadowaki E, Sato Y, Kajita E, Kagamimori $\mathrm{S}$, et al. Fracture risk prediction using FRAX: a 10-year follow-up survey of the Japanese Population-Based Osteoporosis (JPOS) Cohort Study. Osteoporos Int 2011;22:3037-45.

31. Cheung EY, Bow CH, Cheung CL, Soong C, Yeung S, Loong C, et al. Discriminative value of FRAX for fracture prediction in a cohort of Chinese postmenopausal women. Osteoporos Int 2012;23:871-8.

32. Sandhu SK, Nguyen ND, Center JR, Pocock NA, Eisman JA, Nguyen TV. Prognosis of fracture: evaluation of predictive accuracy of the FRAX algorithm and Garvan nomogram. Osteoporos Int 2010;21:863-71.

33. Lee DY, Lim SJ, Moon YW, Min YK, Choi D, Yoon BK, et al. Determination of an applicable FRAX model in Korean women. J Korean Med Sci 2010;25:1657-60.

34. McCloskey EV, Oden A, Harvey NC, Leslie WD, Hans D, Johansson $\mathrm{H}$, et al. Adjusting fracture probability by trabecular bone score. Calcif Tissue Int 2015;96:500-9.

35. Schousboe JT, Vo T, Taylor BC, Cawthon PM, Schwartz AV, Bauer DC, et al. Prediction of incident major osteoporotic and hip fractures by trabecular bone score (TBS) and prevalent radiographic vertebral fracture in older men. J Bone Miner Res 2016;31:690-7.

36. Ripamonti C, Lisi L, Buffa A, Gnudi S, Caudarella R. The trabecular bone score predicts spine fragility fractures in postmenopausal Caucasian women without osteoporosis independently of bone mineral density. Med Arch 2018;72:46-50.

37. Mirzaei A, Jahed SA, Nojomi M, Rajaei A, Zabihiyeganeh M. A study of the value of trabecular bone score in fracture risk assessment of postmenopausal women. Taiwan J Obstet Gynecol 2018;57:389-93.

Copyright (C) 2020 Korean Endocrine Society 